\title{
Chapter 3. What is a high-risk group for CKD?
}

- Risk factors for the development of CKD are: aging, family history of CKD, habitual user of non-steroidal anti-inflammatory drugs (NSAIDs), history of abnormal urine findings, abnormal kidney function, abnormal morphology of kidney or acute kidney injury, dyslipidemia, hyperuricemia, hypertension, impaired glucose tolerance or diabetes mellitus, obesity, metabolic syndrome, collagen disease, infectious disease, and nephrolithiasis.

- As a safeguard against the development of CKD, hypertension and diabetes must be kept under control in individuals belonging to these high-risk groups, and their lifestyle should also be modified.

- One of the most important causal factors of kidney function deterioration in healthy people is aging. The degrees of the decline vary considerably among individuals. Risk factors for atherosclerosis, which are associated with hypertension, diabetes, obesity, and dyslipidemia, increase with aging. Once the glomerular filtration rate (GFR) decreases, anemia, hypertension, proteinuria and abnormal electrolyte metabolism are more likely to appear, further accelerating the decline in GFR.

- Results from health examination demonstrate that risk factors for development of stages 1-2 CKD (positive for proteinuria) during a 10-year follow-up period are age, hematuria, hypertension, and impaired glucose tolerance (IGT) (Fig. 3-1). Those for developing stages 3-5 CKD (eGFR $\left.<60 \mathrm{~mL} / \mathrm{min} / 1.73 \mathrm{~m}^{2}\right)$ include age, proteinuria, hematuria/proteinuria, hypertension, longterm diabetes, dyslipidemia, and smoking (Fig. 3-2). These results suggest that it is particularly necessary for individuals who belong to a high-risk group to quit smoking and treat hypertension, IGT/diabetes, dyslipidemia, and obesity to prevent the development of CKD.
Males have been shown to develop proteinuria more often than females and therefore should be put on stricter treatment regimens and be required to modify their lifestyle.

- For a case with a family history of CKD, lifestyle modifications, such as stopping with smoking and a low salt diet, is recommended. For individuals with abnormal urine findings at a recent health examination, kidney dysfunction, abnormal morphology of the kidney, habitual intake of drugs, such as NSAIDs, or acute kidney injury, modifications in lifestyle are encouraged, and regular follow-up examinations of kidney function and urine tests are needed to detect CKD at an earlier stage.

- Hypertension is a treatable risk factor in many cases and should be adequately managed in a high-risk group of CKD. The higher the blood pressure, the greater the risk of proteinuria and the higher the incidence of endstage kidney disease (ESKD). Adequate control of blood pressure is one of the most effective approaches to managing CKD.

- Although diabetic nephropathy is the leading cause of ESKD in Japan, adequate control of the blood glucose level may prevent the development of CKD or improve the severity (stage). The Kumamoto Study and UKPDS suggest that a good control of blood glucose prevents diabetic nephropathy. It is noted that pancreas transplantation improves diabetic nephropathy.

- Obesity is a significant risk factor for proteinuria and ESKD development, especially in males.

- Dyslipidemia is a risk factor of atherosclerosis. Although based on very little evidence, it has been suggested that a complication of dyslipidemia may promote ESKD. Increases in urinary protein excretion are associated with increased incidence of dyslipidemia. 
Fig 3-1 Risk factors for the development of stages 1-2 chronic kidney disease. GFR Glomerular filtration rate, $D M$ diabetes mellitus. The data are quoted, with modification, from: Yamagata $\mathrm{K}$ et al. (Kidney Int. 2007;71:159-166)
Fig. 3-2 Risk factors for the development of stages 3-5 CKD. HDL High-density lipoprotein. The data are quoted, with modification, from:

Yamagata $\mathrm{K}$ et al. (Kidney Int. 2007;71:159-166)

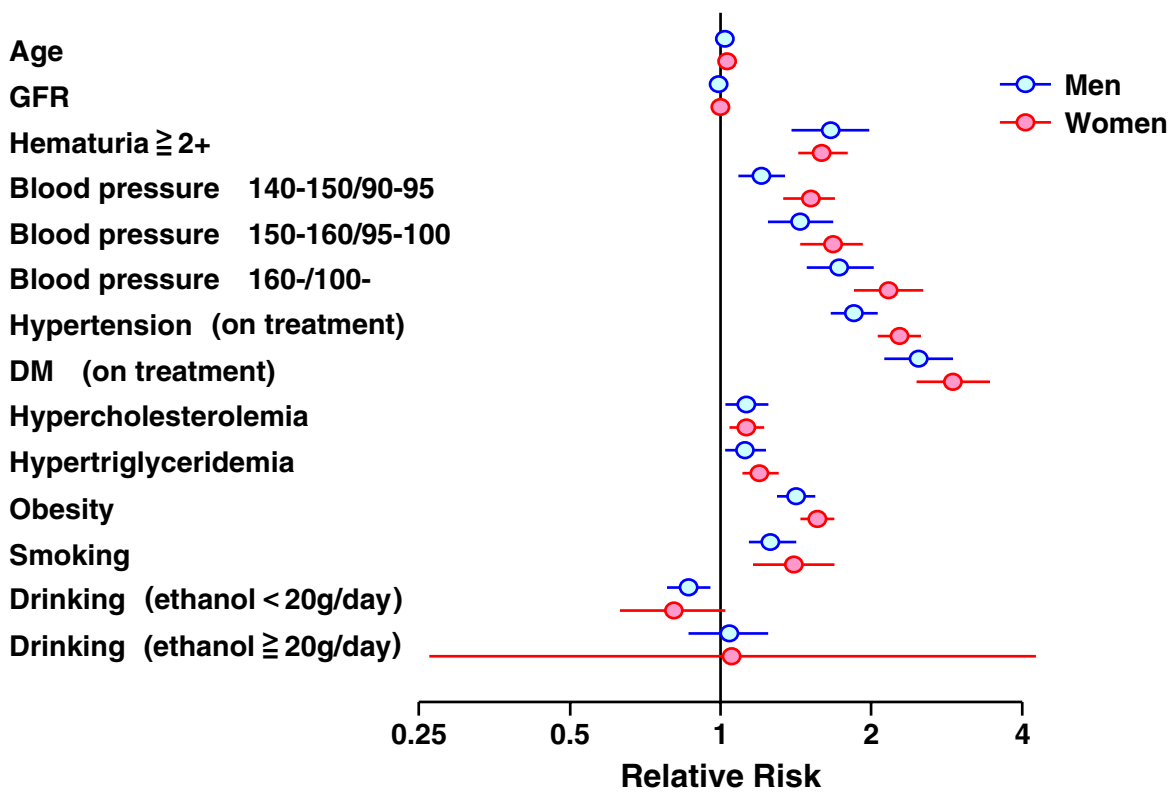

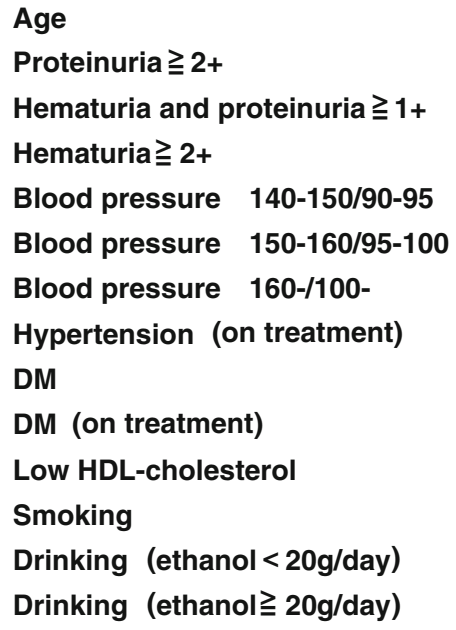

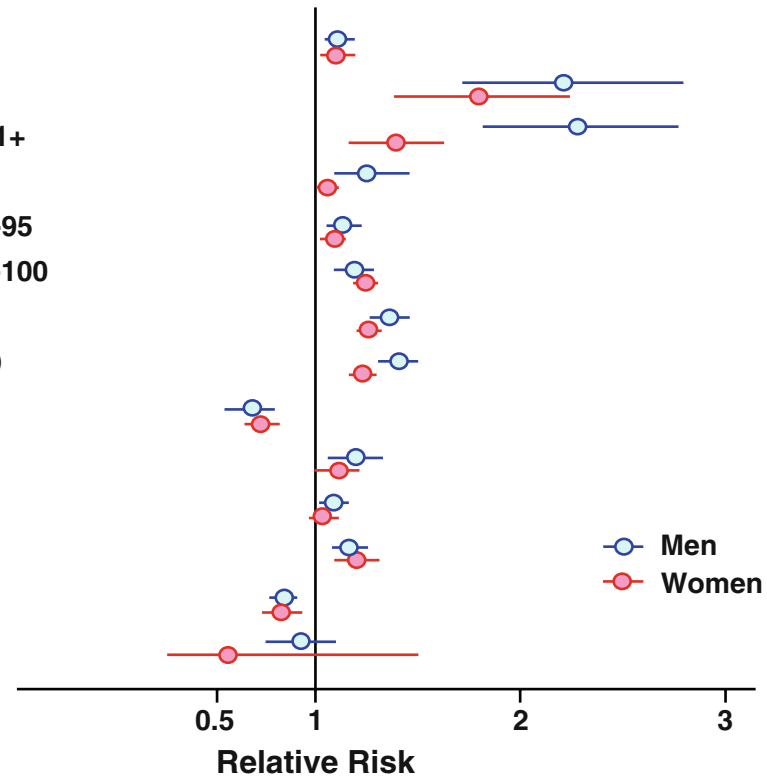

tor for atherosclerosis, since hyperuricemic patients have hypertension and other risk factors for atherosclerosis. 